a principal of integration with the relevant end-users of the research findings is of paramount importance. Mechanisms which enable capture of end-users' preferences for mode, timing, content and ways for seeking feedback around dissemination/engagement strategies should be in place. In addition to this, communicating/engaging with members of the public requires careful consideration of the targeted audience and mode of delivery. Lack of time, resources, training and support (both at institutional and senior staff level) hinders the development of good dissemination/ engagement strategies.

Conclusions Alongside time and resources, support and cultural shifts are needed to allow a full implementation of the principles of a good dissemination/engagement strategy.

\section{WHAT'S IN A NAME? THE INTENSIVE AND PALLIATIVE CARE UNIT}

Guy Parsons. Oxford University and Thames Valley Deanery, UK

10.1136/spcare-2019-mariecuriepalliativecare.40

Death in the Intensive Care Unit (ICU) is common (Angus 2004) and critical care admissions in the last month of life are becoming more frequent. (Teno 2013) Traditional views on the ICU as a place to only support 'good candidates' with deranged physiology while awaiting resolution of underlying pathology appear to be changing. An ageing and increasingly co-morbid population, alongside medical and surgical advances, have seen ICUs admit older and sicker patients year on year. Determining which patients are likely to die or to survive can be fraught with difficulty, especially early into admissions. Prognostic models, using populationlevel data, are fallible and reduce poorly to the individual level. As admissions progress clinical opinion constellates on clearer views of patients' trajectories however it is likely that most will have been experiencing severe and distressing symptoms for some time.(Puntillo 2010) Relatives are also profoundly affected and represent a major area of clinical interaction and discussion.(McAdam 2010).

While it may initially appear that Intensive Care and Palliative Care clinicians have divergent aims, the amelioration of distressing symptoms and focus on quality of life are in fact shared goals. What is lacking is a truly shared space to address these aims. It is time to formally acknowledge the value of bringing the Hospice to the ICU, grasping the value overlapping skillsets can bring, and anchoring the goal of bringing quality to life and dignity to death in a new name: The Intensive and Palliative Care Unit.

\section{FROM PAPER TO PAPERLESS: DO ELECTRONIC SYSTEMS ENSURE SAFE AND EFFECTIVE COMMUNICATION AND DOCUMENTATION OF DO NOT ATTEMPT CARDIO- PULMONARY RESUSCITATION (DNACPR) DECISIONS?}

Laura Harrington, Kathryn Price, Polly Edmonds. King's College Hospitals NHS Foundation Trust, London, UK

10.1136/spcare-2019-mariecuriepalliativecare.41

Introduction and aim With NHS Trusts making the transition from paper to paperless; it is vital that integration of electronic systems are safe for patients. Following introduction of an electronic-DNACPR toolbar, this project aimed to improve the quality of documentation and communication of DNACPR decisions.

Method A singled centered, closed loop quality improvement project was performed January - June 2018 including all inpatients with a DNACPR on three gerontology wards. Data was collected over a four week period. The DNACPR toolbar, eform, medical notes and discharge letters were reviewed. The documentation of discussions with patients, families, senior doctors, nursing staff and the MDT were recorded. Retrospective discharge analysis was performed to review communication to the GP. Chi Squared analysis was used to assess statistical significance of the raw measure improvements after the second cycle.

Interventions (Figure 1)

Results (Figure 2)

January cycle, all 181 patients had a resuscitation status and treatment escalation plan (TEP). $73 \%$ had a DNACPR ( $86 \%$ by senior doctor). $85 \%$ had a valid e-form. $68 \%$ evidenced a discussion patients/relatives, $13 \%$ had a documented discussion with the MDT. $79 \%$ were communicated on discharge through comprehensive geriatric assessment (CGA). Following interventions, all measures improved. Of 176 patients; $72 \%$ a DNACPR decision (93\% by senior doctor), $100 \%$ an e-form. $71 \%$ evidenced discussion with patients/relatives, $57 \%$ had documented discussion with the MDT.

Conclusion A critical finding was poor communication, increasing risk of inappropriate resuscitation. Process revision and staff training has led to improved communication and documentation, increasing patient safety. DNACPR decision inclusion in discharge letters via CGAs has improved care coordination on discharge.

\section{EVALUATING CHANGES TO THE WAY PSYCHOLOGICAL SERVICES ARE DELIVERED WITHIN A HOSPICE}

${ }^{1}$ Nick Hartley, ${ }^{2}$ Gareth Luce. ${ }^{1}$ Newcastle upon Tyne Hospitals NHS Trust, Newcastle upon Tyne, UK; ${ }^{2}$ University of Newcastle, Newcastle upon Tyne, UK

\subsection{6/spcare-2019-mariecuriepalliativecare.42}

Background There is a growing evidence base for the role of Clinical Psychology within Palliative Care. To improve provision within their local hospice, Newcastle upon Tyne Hospitals NHS Trust secured an agreement with Marie Curie for funding a Clinical Psychologist to be physically based at the local hospice for two days a week. This change in service has now been provided since July 2017.

Aims and objectives The project evaluates the impact of having a Clinical Psychologist onsite at the hospice, I comparison to the previous service provision from the wider Specialist Palliative Care Clinical Psychology service that provides input to the two hospitals, hospices and community palliative care team from a base in the community. The project was completed with a view to informing future service development.

Summary of project Healthcare professionals at Marie Curie Hospice were interviewed about their experiences of this change in provision, with respect to the benchmarks for quality care defined by the British Psychological Society. A thematic analysis of these interviews was then conducted to provide a descriptive account of staff perceptions. 